\title{
Constraints on millicharged particles from cosmic-ray production
}

\author{
Ryan Plestid $\odot,{ }^{1,2,3,4, *}$ Volodymyr Takhistov, ${ }^{5, \dagger}$ Yu-Dai Tsai $\odot,{ }^{2,6, *}$ Torsten Bringmann $\odot,{ }^{7,8}$ \\ Alexander Kusenko, ${ }^{5,8, \|}$ and Maxim Pospelov ${ }^{9,10, \pi}$ \\ ${ }^{1}$ Department of Physics and Astronomy, University of Kentucky, Lexington, Kentucky 40506, USA \\ ${ }^{2}$ Theoretical Physics Department, Fermilab, Batavia, Illinois 60510, USA \\ ${ }^{3}$ Perimeter Institute for Theoretical Physics, 31 Caroline Street North, \\ Waterloo, Ontario N2L 2Y5, Canada \\ ${ }^{4}$ Department of Physics and Astronomy, McMaster University, \\ 1280 Main Street West, Hamilton, Ontario L8S 4M1, Canada \\ ${ }^{5}$ Department of Physics and Astronomy, University of California, \\ Los Angeles, California 90095-1547, USA \\ ${ }^{6}$ Cosmic Physics Center, Fermi National Accelerator Laboratory, Batavia, Illinois 60510, USA \\ ${ }^{7}$ Department of Physics, University of Oslo, Box 1048, N-0371 Oslo, Norway \\ ${ }^{8}$ Kavli Institute for the Physics and Mathematics of the Universe (WPI), \\ UTIAS The University of Tokyo, Kashiwa, Chiba 277-8583, Japan \\ ${ }^{9}$ School of Physics and Astronomy, University of Minnesota, Minneapolis, Minnesota 55455, USA \\ ${ }^{10}$ William I. Fine Theoretical Physics Institute, School of Physics and Astronomy, \\ University of Minnesota, Minneapolis, Minnesota 55455, USA
}

(Received 13 March 2020; accepted 7 December 2020; published 24 December 2020)

\begin{abstract}
We study cosmic-ray-atmosphere collisions as a permanent production source of exotic millicharged particles (MCPs) for all terrestrial experiments. [MCPs are also known as charged massive particles (CHAMPs).] Based on data from Super-K, this allows us to derive new limits on MCPs that are competitive with, or improve, the currently leading bounds from accelerator-based searches for masses up to $1.5 \mathrm{GeV}$. In models where a subdominant component of dark matter (DM) is fractionally charged, these constraints probe parts of the parameter space that is inaccessible for conventional direct-detection DM experiments, independently of assumptions about the DM abundance.
\end{abstract}

DOI: 10.1103/PhysRevD.102.115032

\section{INTRODUCTION}

The remarkable success of the Standard Model (SM), along with null results at the LHC, suggests that if new physics exists below the $\mathrm{TeV}$ scale it must be weakly coupled to SM degrees of freedom. Such a dark sector would likely leave its strongest imprint on SM degrees of freedom commensurate with its own dynamical energy scales [1]. The $\mathrm{MeV}-\mathrm{GeV}$ regime both contains many SM particles and hosts a number of persistent anomalies, including the anomalous magnetic moment of the muon

*rpl225@uky.edu

vtakhist@physics.ucla.edu

†ytsai@fnal.gov

\$torsten.bringmann@fys.uio.no

"kusenko@ucla.edu

"mpospelov@perimeterinstitute.ca

Published by the American Physical Society under the terms of the Creative Commons Attribution 4.0 International license. Further distribution of this work must maintain attribution to the author(s) and the published article's title, journal citation, and DOI. Funded by SCOAP ${ }^{3}$.
[2-4]. At these energies, new physics can be efficiently probed by fixed-target experiments [5-9] with high intensity electron $[10,11]$ and proton beams [12-20] where dark sector particles can be produced either directly, or through meson decays; collider experiments typically provide the leading constraints at higher masses [21-23]. A less explored opportunity is the production of dark sector particles in cosmic-ray interactions and their subsequent detection in large detectors [24-34].

Historically, the discovery of particles in the MeV-GeV regime (e.g., pions [35] and muons [36]) often resulted from cosmic rays, mostly protons, bombarding the upper atmosphere. This essentially constitutes a fixed-target experiment, where the proton "beam" is always on. With modern neutrino telescopes, the detectors located "downstream" can be kton-Mton scale (e.g., IceCube [37], SuperK [38], Hyper-K [39], JUNO [40], DUNE [41]) and can serve as a powerful tool with which to probe the dark sector.

In this work, we calculate a lower bound on the flux of millicharged particles (MCPs) [42-48], $\chi$, arising from meson decays in the upper atmosphere for $m_{\chi}$ in the $\sim 100 \mathrm{MeV}$ to few $\mathrm{GeV}$ regime. For this, we adopt a 
minimal model about the new particle that is based on only two assumptions which are as follows:

(1) $\chi$ couples to the photon with strength $Q_{\chi}=\epsilon \times e$; we remain agnostic as to the origin of this charge.

(2) $\chi$ is stable, which is a natural consequence if $Q_{\chi}$ is the smallest (nonzero) charge in the dark sector.

Our analysis then serves as a benchmark for the sensitivity of neutrino observatories to stable dark sector particles that can be produced in the upper atmosphere. In particular, our constraints apply (possibly conservatively) to any model that satisfies the above two assumptions.

One immediate consequence of our calculation is that existing constraints on an ambient ionizing MCP fluxquoted as a function of $\epsilon$ and $\Phi_{\text {ion }}(\epsilon)$-from MACRO [49,50], Kamiokande-II [51], LSD [52], CDMS [53], and Majorana [54] can now for the first time be recast as direct constraints on $\epsilon$ as a function of $m_{\chi}$. Better yet, we find that neutrino observatories can set new competitive (and in some cases leading) bounds on MCP couplings in the $100-500 \mathrm{MeV}$ range based on existing data, surpassing the reach of fixed-target experiments with neutrino detectors. We demonstrate this point explicitly by providing novel constraints based on published analyses by the SuperKamiokande (Super-K, SK) Collaboration searching for the diffuse supernova neutrino background (DSNB) [55]. Our results, summarized in Fig. 1, suggest that future neutrino telescopes could be able to act as a robust, and in

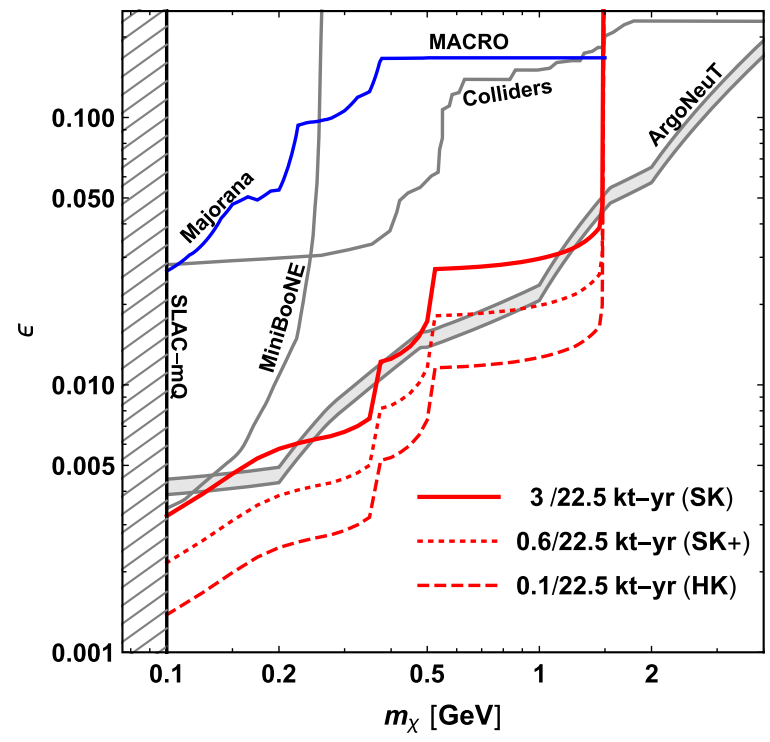

FIG. 1. Exclusion limits for MCPs from cosmic-ray interactions (SK, red solid), based on the diffuse supernova neutrino background search in Super-K [55], as well as sensitivity projections for an upgrade with gadolinium doping ( $\mathrm{SK}+$, red dotted) and near-future Hyper-K (HK, red dashed). We also display new limits (blue) from recasting data of MACRO [49,50] and Majorana [54]. Previous limits from electron fixed-target (SLAC MilliQ [10,11], MiniBooNE [16,60], ArgoNeuT [61]) and collider experiments [6,62-64] (as compiled in Ref. [61]) are shown for comparison. fact the leading, probe of MCPs in the $100 \mathrm{MeV}-1.5 \mathrm{GeV}$ regime, free from cosmological assumptions. Our results apply to MCP parameter space important to $21 \mathrm{~cm}$ cosmology (potentially explaining the EDGES anomaly [56-59]) and to the same regime that has motivated the proposal of dedicated detectors such as MilliQan [22,23] and FerMINI [18].

More broadly, we note that MCPs have connections to charge quantization, which is itself connected to, but does not necessarily preclude [65], the existence of magnetic monopoles [66], grand unification [67-70], and quantum gravity [71]. Furthermore, they appear naturally in models of light dark matter (DM) [65,72-74], and a fast-moving flux of MCPs has recently been proposed to explain a reported excess in direct-detection experiments $[75,76]$.

\section{STRONGLY INTERACTING DM}

Our results can be recast as limits on millicharged strongly interacting DM (SIDM) [77,78]. This refers to a class of models where the DM-SM particle (mostly nuclei and electrons) cross section is so large that the DM flux reaching conventional underground direct-detection experiments would be significantly attenuated, thus preventing detection [77,79]. Therefore, above some critical value of $\epsilon$ fractionally charged DM remains unconstrained by such experiments [78], and there is a window of available parameter space where MCPs could constitute a subdominant component of DM $(\lesssim 0.4 \%$ to avoid cosmological constraints [80-83]); we will refer to this as the millicharged SIDM window (see Fig. 2). New balloon and satellite experiments have been proposed [78] to further explore this window, which could accommodate DM models capable of explaining the EDGES anomaly [56-59,84].

Our results exclude parameter space for millicharged SIDM that is not accessible at terrestrial detectors because the typical velocity of MCPs produced by cosmic rays is much higher than the ambient dark matter wind, such that cosmic-ray-produced MCPs can reach subterranean detectors with enough kinetic energy to leave detectable signatures. In Fig. 2, we translate our results from Fig. 1 to constraints on a conventional "reference cross section" $\bar{\sigma}_{e, \text { ref }}=16 \pi \alpha^{2} \epsilon^{2} \mu_{\chi e}^{2} / q_{\mathrm{d}, \text { ref }}^{4}$ for DM-electron scattering ${ }^{1}$ (which for light MCPs dominates over scattering with nuclei). Here, $\mu_{\chi e}$ is the reduced mass of the electron and $\chi$ and $q_{d \text {,ref }}$ are the typical momentum transfer in $\chi-e$ scatterings for semiconductor or noble-liquid targets, taken to be $\alpha m_{e}$ [78]. In the same figure, we compare to existing

\footnotetext{
${ }^{1}$ In Ref. [78], the DM millicharge is generated through a coupling to a massless dark photon that kinetically mixes with the SM $U(1)_{Y}$; here we directly consider the DM to have a millicharge under $U(1)_{\mathrm{Y}}$, with minimal theoretical assumptions about the origin of this charge. The reference cross section we consider corresponds to $\alpha_{D}=\alpha$ and $m_{A}^{\prime} \rightarrow 0$ in Eq. (2.6) of Ref. [78].
} 


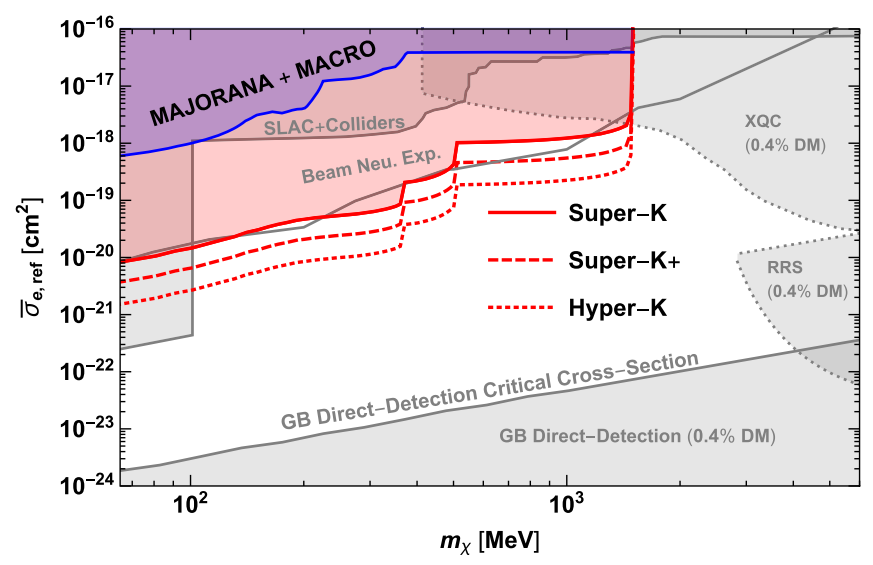

FIG. 2. Constraints and sensitivity reaches around the millicharged SIDM window, including our new bounds from recasting Super-K data [55] (red) and projections for a Super-K upgrade (red, dashed) and Hyper-K (red, dotted). We also show new bounds (blue) from recasting data of MACRO [49,50] and Majorana [54], along with existing accelerator-based constraints [6,10,11,16,61-64], limits from the above-atmosphere detector (RRS) [85], a rocket experiment (XQC) [86,87], and groundbased direct detection [78].

constraints, but do not consider here bounds based on the $\mathrm{MCP}$ acceleration from astrophysical sources that rely on additional assumptions beyond the local DM density $[26,47,48,88]$. Our constraints are independent of the fractional composition of DM [80-82], since our millicharged particles are directly produced in the upper atmosphere; for reference cross sections below approximately $10^{-17} \mathrm{~cm}^{2}$, our results are insensitive to attenuation in the Earth.

\section{COSMIC-RAY MESON PRODUCTION}

We begin by discussing the production of mesons from cosmic rays, which then subsequently decay to MCPs. Cosmic rays hitting the upper atmosphere dominantly consist of high-energy protons and are isotropically distributed. Their flux is typically quoted in terms of intensity, $\left[\mathcal{I}_{\mathrm{CR}}\right]=\mathrm{GeV}^{-1} \mathrm{~cm}^{-2} \mathrm{~s}^{-1} \mathrm{str}^{-1}$ [89], which in our analysis we take from DarkSUSY [90] (based on Ref. [91]).

Taking into account that all incoming cosmic rays are eventually absorbed by the atmosphere, we can treat it as a "thick target." In what follows, we (i) treat $p A$ collisions as an incoherent sum of $p p$ collisions and (ii) we include only primary production. With these two assumptions, we can calculate the yield of primary mesons per incident proton as $\sigma_{\mathfrak{m}} / \sigma_{\text {in }}$ where $\sigma_{\mathfrak{m}}$ is the inclusive $p p \rightarrow \mathfrak{m} X$ production cross section, and $\sigma_{\text {in }}$ is the total $p p$ inelastic cross section. This provides a robust lower bound on the flux of mesons produced by cosmic rays in the upper atmosphere. We take $\sigma_{\text {in }}(p p)$ from Ref. [89], expressed in terms of the center-of-mass boost $\gamma_{\mathrm{cm}}=$ $\frac{1}{2} \sqrt{s} / m_{p}$ and $\sigma_{\mathfrak{m}}$ from a number of accelerator-based experiments. $^{2}$ We focus on the dominant $\eta$, light vector, and $J / \psi$ mesons. While we have also quantitatively considered $\Upsilon$ meson as well as direct Drell-Yan production, we found these contributions to be negligibly small (6 orders of magnitude smaller than $J / \psi$ ). We focus our attention on $m_{\chi} \gtrsim 100 \mathrm{MeV}$ due to strong existing constraints from a combination of SLAC's milliQ experiment [10] and LSND's search for electronlike scattering events [102]. This eliminates $\pi^{0}$ as a potential source of MCPs. The resulting meson flux from cosmic-ray collisions in the upper atmosphere is then given by

$\Phi_{\mathfrak{m}}\left(\gamma_{\mathfrak{m}}\right)=\Omega_{\mathrm{eff}} \int \mathcal{I}_{\mathrm{CR}}\left(\gamma_{\mathrm{cm}}\right) \frac{\sigma_{\mathfrak{m}}\left(\gamma_{\mathrm{cm}}\right)}{\sigma_{\mathrm{in}}\left(\gamma_{\mathrm{cm}}\right)} P\left(\gamma_{\mathfrak{m}} \mid \gamma_{\mathrm{cm}}\right) \mathrm{d} \gamma_{\mathrm{cm}}$,

where $\mathcal{I}_{\mathrm{CR}}\left(\gamma_{\mathrm{cm}}\right) \equiv \mathcal{I}_{\mathrm{CR}}\left(E_{p}\right) \times \mathrm{d} E_{p} / \mathrm{d} \gamma_{\mathrm{cm}}$ and $\Omega_{\text {eff }} \approx 2 \pi$ is the effective solid angle from which MCPs can arrive at the detector, ${ }^{3}$ and $P\left(\gamma_{\mathfrak{m}} \mid \gamma_{\mathrm{cm}}\right)$ represents the probability to get a meson with boost $\gamma_{\mathfrak{m}}$ in the lab frame. We emphasize that the primary yield per proton $\sigma_{\mathfrak{m}} / \sigma_{\text {in }}$ and the cosmic-ray intensity $\mathcal{I}$ are well measured (see Appendix B) and determine the total integrated flux of mesons. In contrast, $P\left(\gamma_{\mathfrak{m}} \mid \gamma_{\mathrm{cm}}\right)$ does not affect the normalization, only serving to redistribute the flux as a function of $\gamma_{\mathfrak{m}}$. This probability can be conveniently estimated (see Appendix B) from the differential production cross section with respect to $x_{F} \equiv p_{L} / p_{\max }$, where $p_{L}$ is the longitudinal momentum and $p_{\max }$ is the maximum possible momentum,

$$
P\left(\gamma_{\mathfrak{m}} \mid \gamma_{\mathrm{cm}}\right) \approx \sum_{\alpha} \frac{1}{\sigma_{\mathfrak{m}}} \times \frac{\mathrm{d} \sigma_{\mathfrak{m}}}{\mathrm{d} x_{F}} \times \frac{\mathrm{d} x_{F}^{(\alpha)}}{\mathrm{d} \gamma_{\mathfrak{m}}}
$$

Here $\alpha= \pm$ denotes the two different branches that appear when inverting $\gamma_{\mathfrak{m}}\left(x_{F}\right)$, and $\mathrm{d} \sigma_{\mathfrak{m}} / \mathrm{d} x_{F}$ is a function of $\gamma_{\mathrm{cm}}$ and $x_{F}\left(\gamma_{\mathrm{m}}\right)$. The meson-production energy spectrum thus depends on both the total meson cross section, $\sigma_{\mathfrak{m}}\left(\gamma_{\mathrm{cm}}\right)$, and the differential cross section with respect to $x_{F}$, or equivalently on $P\left(\gamma_{\mathrm{m}} \mid \gamma_{\mathrm{cm}}\right)$. Our parametrization of $\mathrm{d} \sigma_{\mathfrak{m}} / \mathrm{d} x_{F}$ is guided by empirical measurements, but, with the exception of $J / \psi$, data on the $x_{F}$ distribution are limited to specific center-of-mass energies and we adopt a prescription outlined in Appendix B. The normalization of the flux is independent of this prescription, and the shape of the resultant meson fluxes depends only weakly on it.

\footnotetext{
${ }^{2}$ For $\eta$, see Refs. [92-97]. For $\rho, \omega$, and $\phi$, see Refs. [94,95,97]. Finally, for $J / \psi$, see Refs. [98-101]. Details of the analysis are discussed in Appendix B.

${ }^{3}$ By rescaling the muon's stopping power $[26,89]$, we estimate that the energy loss of MCPs in the Earth's crust (standard rock) is roughly $50 \mathrm{MeV} / \mathrm{km}$ for $\epsilon \sim 10^{-2}$. While for the range of $\epsilon$ and energies that we are interested in here, MCPs interact too strongly to penetrate the entire Earth, they are not significantly impeded to reach the detector when originating from the upper hemisphere.
} 


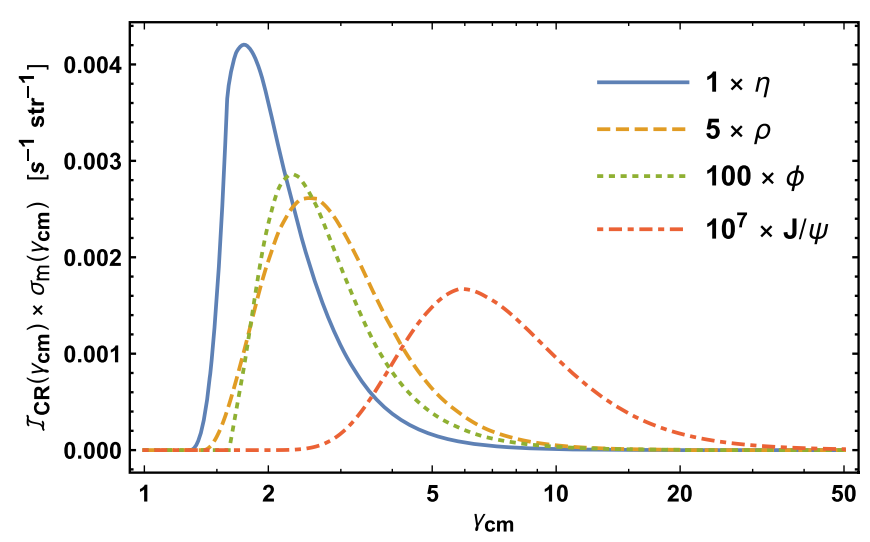

FIG. 3. Differential cosmic-ray intensity, $\mathcal{I}_{\mathrm{CR}}$, multiplied by the yield per proton $\sigma_{\mathrm{m}} / \sigma_{\text {in }}$ as a function of $\gamma_{\mathrm{cm}}$. For the resulting meson spectra, see Appendix E.

In Fig. 3, we display the differential cosmic-ray intensity $I_{\mathrm{CR}}\left(\gamma_{\mathrm{cm}}\right)$ multiplied by yield per proton $\sigma_{\mathfrak{m}}\left(\gamma_{\mathrm{cm}}\right)$ for each meson species. This plot shows us which parts of the primary cosmic-ray spectrum are responsible for each meson's (primary) production; we emphasize again that this results in a robust lower bound on the flux of mesons from the upper atmosphere computed from microscopic considerations. The shape of these curves is determined by the competition between a rising inclusive cross section and a sharply falling cosmic-ray flux and illustrates which parts of the cosmic-ray spectrum predominantly contribute to a given meson species' flux.

\section{MCP FLUX FROM MESON DECAYS}

Upon constructing $\Phi_{\mathfrak{m}}$ as outlined above, we find the associated flux of MCPs from meson decays by folding the meson flux with the unit-normalized spectrum of MCPs in the lab frame, $P\left(\gamma_{\chi} \mid \gamma_{\mathfrak{m}}\right)$, and weighting by the decay branching ratio, Meson kinematics are discussed in Appendix A

$$
\Phi_{\chi}\left(\gamma_{\chi}\right)=2 \sum_{\mathfrak{m}} \mathrm{BR}_{\mathfrak{m} \rightarrow \chi \bar{\chi}} \int \mathrm{d} \gamma_{\mathfrak{m}} \Phi_{\mathfrak{m}}\left(\gamma_{\mathfrak{m}}\right) P\left(\gamma_{\chi} \mid \gamma_{\mathfrak{m}}\right)
$$

where the factor of 2 accounts for the contribution from both $\bar{\chi}$ and $\chi$. The quantity $P\left(\gamma_{\chi} \mid \gamma_{\mathfrak{m}}\right)=\left[\Gamma^{-1} \mathrm{~d} \Gamma / \mathrm{d} \gamma_{\chi}\right]_{\text {lab }}$ can be calculated from first principles, at leading order in $\epsilon$, where $\Gamma$ is the decay rate for $\mathfrak{m} \rightarrow \chi \bar{\chi}$ and $\mathrm{d} \Gamma / \mathrm{d} \gamma_{\chi}$ is the differential rate with respect to the MCP boost, both evaluated in the lab frame, Appendix B.

We define the integrated "fast flux" of MCPs satisfying $\gamma_{\chi} \geq \gamma_{\text {cut }}$ as

$$
\Phi_{\text {cut }}\left(m_{\chi}, \gamma_{\text {cut }}\right)=\int_{\gamma_{\text {cut }}}^{\infty} \mathrm{d} \gamma_{\chi} \frac{\mathrm{d} \Phi_{\chi}}{\mathrm{d} \gamma_{\chi}}
$$

where $\gamma_{\text {cut }}$ is set by the relevant experimental threshold. In Fig. 4, we display this quantity's mass dependence for

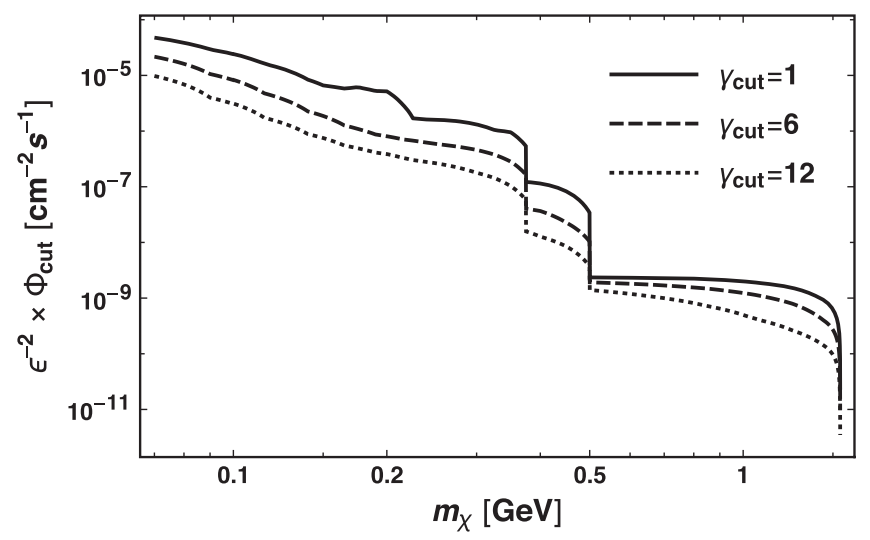

FIG. 4. Fast flux of MCPs due to meson decays, $\Phi_{\text {cut }}$, as a function of the MCP mass, $m_{\chi}$, for three different choices of $\gamma_{\text {cut }}$. The full integrated MCP flux is obtained for $\gamma_{\text {cut }}=1$. The meson mass thresholds are clearly visible, stemming from $\eta, \omega / \rho, \phi$, and finally $J / \psi$ (sequentially from left to right).

several choices of $\gamma_{\text {cut }}: \gamma_{\text {cut }}=1$ i.e., the full integrated MCP flux relevant for ionization experiments, $\gamma_{\text {cut }}=6$ as required for an electron recoil of $T_{\min }=16 \mathrm{MeV}$ (relevant for Super-K), and $\gamma_{\text {cut }}=12$ for illustration.

\section{DETECTING MCPS IN LABORATORIES}

Our calculation of the flux as a function of $\epsilon$ and $m_{\chi}$ allows us to derive constraints on $\epsilon$ as a function of $m_{\chi}$. For the case of ionization experiments, we can directly compare the $\gamma_{\text {cut }}=1$ curve in Fig. 4 to published bounds in terms of the ambient MCP flux (see Appendix C for details), and our results establish a quantitative baseline that can be used to estimate the impact of upcoming projects such as LEGEND [103]. For MCPs with large charges of $\epsilon \gtrsim 10^{-1}$, effects of attenuation when passing through Earth to reach typical detector depths of $\sim 1 \mathrm{~km}$ of standard rock, i.e., few $\mathrm{km}$ water equivalent, become significant (see e.g., Ref. [26]). We do not consider these effects here as this region is already well constrained by collider searches (which are not sensitive to attenuation).

Electron scattering inside Cherenkov detectors, with recoils in the $\sim 10 \mathrm{MeV}$ range, can also probe MCPs [15] (see also [26]). The detection of MCPs is dominated by soft scattering from electrons as can be readily understood by considering the differential scattering cross section which, being mediated by photon exchange, scales as $\mathrm{d} \sigma / \mathrm{d} Q^{2} \sim 1 / Q^{4}$. For elastic scattering from a target of mass $M$, the momentum transfer is given by $Q^{2}=2 M\left(E^{\prime}-M\right)$, where $E^{\prime}$ is the total recoil energy of the target. The cross section is therefore maximized by scattering off the lightest target possible with the lowest possible recoil energy. In practice, experimental considerations such as detection efficiency and background reduction will set a minimum electron recoil energy which will, in turn, dictate the detection cross section for that 
given experiment. We will therefore consider a windowed cross section for electron recoils with kinetic energy, $T_{e}^{\prime}=$ $\left(E_{e}^{\prime}-m_{e}\right)$ between $T_{\min }$ and $T_{\max }$, or equivalently with momentum transfers between $Q_{\min }^{2}$ and $Q_{\max }^{2}$,

$$
\tilde{\sigma}_{e \chi}=\int_{Q_{\min }^{2}}^{Q_{\max }^{2}} \frac{\mathrm{d} \sigma_{e \chi}}{\mathrm{d} Q^{2}} \mathrm{~d} Q^{2} .
$$

Since the four-momentum transfer is directly related to the recoil energy in the lab frame, $T_{e}^{\prime}=E_{e}^{\prime}-m_{e}$, via $Q^{2}=-2\left(p_{e}-p_{e}^{\prime}\right)^{2}=\left(2 m_{e} E_{e}^{\prime}-2 m_{e}^{2}\right)=2 m_{e} T_{e}^{\prime}$, this is equivalent to demanding that $Q^{2} \geq 2 m_{e} T_{\min }$. In the center-of-mass frame, the maximal momentum transfer is given when the scattering is back-to-back such that $Q^{2} \leq 4 \mathcal{P}_{e}^{2}-2 m_{e}^{2}$ where $\mathcal{P}_{e}$ is the electron's momentum in the center-of-mass frame,

$$
\mathcal{P}_{e}=\sqrt{\frac{m_{e}^{4}-2 m_{e}^{2}\left(m_{\chi}^{2}+s\right)+\left(m_{\chi}^{2}-s\right)^{2}}{4 s}} .
$$

In terms of lab-frame variables, this implies that

$$
T_{e}^{\prime} \leq \frac{2 m_{e} P_{\chi}^{2}}{2 m_{e} E_{\chi}+m_{e}^{2}+m_{\chi}^{2}} \approx 2 m_{e}\left(\beta_{\chi} \gamma_{\chi}\right)^{2},
$$

where the approximation holds provided $m_{\chi} \gg m_{e} \gamma_{\chi}$. The main consequence of Eq. (7) is that the lower bound of integration in Eq. (5) is given (at leading order in $\left.m_{e} / E_{\chi}\right)^{4}$ by $Q_{\min }^{2}=\max \left(2 m_{e} T_{\min }, 4 m_{e}^{2}\left(\beta_{\chi} \gamma_{\chi}\right)^{2}\right)$, and the upper bound is given by $Q_{\max }^{2}=\min \left(2 m_{e} T_{\max }, 4 m_{e}^{2}\left(\beta_{\chi} \gamma_{\chi}\right)^{2}\right)$. The effect of this approximation on $\tilde{\sigma}_{e \chi}$ can be captured in the approximate expression

$$
\tilde{\sigma}_{e \chi}\left(\gamma_{\chi}\right) \approx \frac{2 \pi \alpha^{2} \epsilon^{2}}{2 T_{\min } m_{e}}\left(1-\frac{T_{\min }}{T_{\max }}\right) \Theta\left(\gamma_{\chi}-\gamma_{\text {cut }}\right),
$$

the accuracy of which is illustrated in Fig. 5. Here, $\alpha$ is the fine-structure constant, $\Theta$ is the Heaviside step function, and $\gamma_{\text {cut }} \approx 0.6 \sqrt{T_{\min } / 2 m_{e}}+0.4 \sqrt{T_{\max } / 2 m_{e}}$. The total resulting number of $\chi-e$ scattering events $N_{e \chi}$ for a given experiment is then

$$
\begin{aligned}
N_{e \chi} & =N_{e} \times t \times \int_{\gamma_{\text {cut }}}^{\infty} \mathrm{d} \gamma_{\chi} \tilde{\sigma}_{e \chi}\left(\gamma_{\chi}\right) \frac{\mathrm{d} \Phi_{\chi}}{\mathrm{d} \gamma_{\chi}}\left(\gamma_{\chi}\right) \\
& \approx N_{e} \times t \times \frac{\pi \alpha^{2} \epsilon^{2}}{T_{\min } m_{e}}\left(1-\frac{T_{\text {min }}}{T_{\text {max }}}\right) \times \Phi_{\text {cut }}\left(m_{\chi}\right),
\end{aligned}
$$

where $N_{e}$ is the number of electrons within the detector's fiducial volume and $t$ is the data collection period.

In summary, the primary driver of the windowed cross section is whether or not the incident MCP is sufficiently boosted to kick the electron above the detection threshold.

\footnotetext{
${ }^{4}$ In producing our exclusion curves, we use the full expression in Eq. (7) rather than the indicated approximation.
}

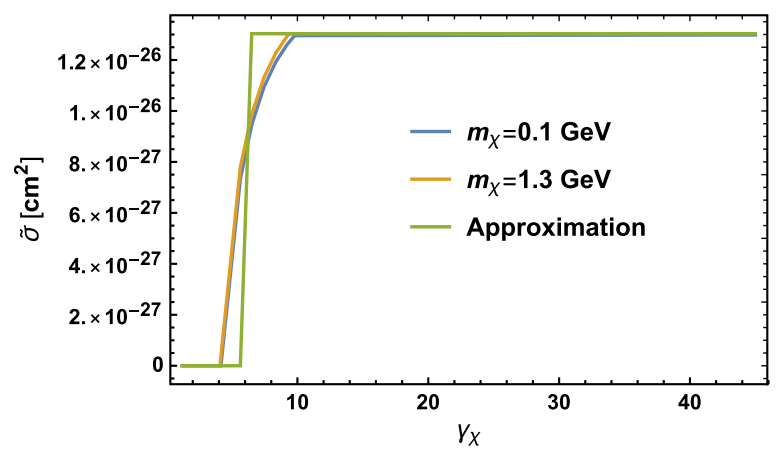

FIG. 5. Dependence of windowed cross section $\tilde{\sigma}_{e \chi}$ on MCP boost factor $\gamma_{\chi}$ for $T_{\min }^{\prime}=16 \mathrm{MeV}$ and $T_{\max }^{\prime}=80 \mathrm{MeV}$ as compared to the approximation Eq. (8).

In principle, the thresholds of large neutrino detectors can be rather low, a few $\mathrm{MeV}$ in case of SK, and as low as $200 \mathrm{keV}$ for Borexino. We choose, however, a much higher threshold of $\sim 15-16 \mathrm{MeV}$, that removes all the events generated by solar neutrinos, so that background counting rates reduce to $O$ (few) per year.

Super-Kamiokande has searched for inverse beta decay positrons [55]. This search looked at inverse beta decays $\bar{\nu}_{e} p \rightarrow n e^{+}$with a positron recoil energy $16 \mathrm{MeV}<T_{e^{+}}<$ $88 \mathrm{MeV}$, corresponding to $\gamma_{\text {cut }} \approx 6$ (Cherenkov detectors cannot differentiate between $e^{-}$and $e^{+}$). Therefore, using the results of the DSNB search in Super-K [55], we can place new limits on MCPs from cosmic-ray production. In the case of ex scattering, the event shape spectrum is determined by the differential cross section with respect to recoil energy, $\mathrm{d} \tilde{\sigma} / \mathrm{d} T_{e} \propto 1 / T_{e}^{2}$, and the incident flux of MCPs. We have confirmed that this shape is very similar to the case of a neutrino spectrum described by temperature of $T_{\nu} \lesssim 5 \mathrm{MeV}$ [55], as shown in Fig. 6, and the results of the likelihood analysis performed in Ref. [55] can therefore be directly employed to constrain the recoil electron spectrum from MCPs. We do not expect that possible coincidence events with MCPs from atmospheric interactions will significantly affect our results, as signal requires significant spatial and temporal correlation which we outline in semiquantitative detail in Appendix D.

We emphasize that the DSNB limits from Super-K are given in terms of limits on the scattered positron event rate as a function of the effective neutrino temperature $T_{\nu}$ from supernova emission. For the reader's convenience, we note that in [55] there are two bounds quoted: one for an ensemble of supernovae of different temperatures and one for a single supernova temperature. We use the latter, because it more closely mimics our signal as is clearly shown in Fig. 6 (the diffuse ensemble would be relatively flat as a function of energy).

We show our resulting bounds on MCPs in Fig. 1, where for the upcoming Super-K upgrade (SK+) with gadolinium doping [104], we assume a year-long exposure and a sensitivity reach of $\sim 0.6$ events $/ 22.5 \mathrm{kt}-\mathrm{yr}$. With a fiducial 


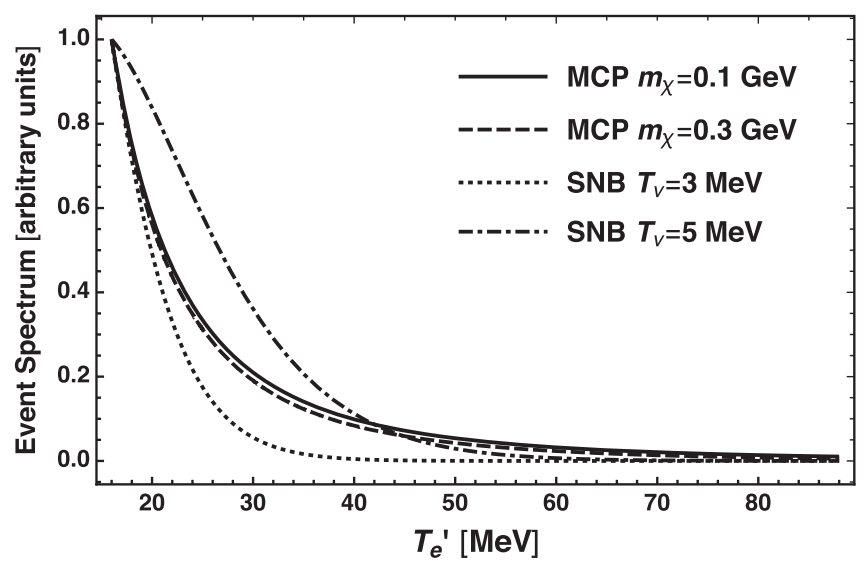

FIG. 6. Comparison of event shapes for MCP elastic scattering off electrons and inverse beta decay from supernova background neutrinos. The MCP signal was obtained by folding the differential scattering cross section $\mathrm{d} \sigma_{e \chi} / \mathrm{d} T_{e}$ against the cosmicray- induced MCP flux. The supernova background curves correspond to $E_{\nu}^{2} /\left(\mathrm{e}^{E_{\nu} / T_{\nu}}+1\right)$ where $E_{\nu}=T_{e}+1.3 \mathrm{MeV}$; these correspond to the fixed temperature profiles used in Ref. [55] as can be readily verified by reproducing their Fig. 19 .

volume of $190 \mathrm{kt}$, the near future Hyper-K water Cherenkov experiment [39], as also shown in this figure, can further improve significantly on these projections. Other near future experiments with sizable fiducial volumes, such as DUNE (40 kton, liquid argon) [41] and JUNO (20 kton, liquid scintillator) [40], will complement water-based Cherenkov detectors as probes of the DSNB [105], and hence can also serve as probes of atmospherically produced MCPs.

\section{SUMMARY}

We have considered MCP production from standard cosmic rays interacting with the atmosphere, constituting a permanent MCP source for all terrestrial experiments. Explicitly calculating the flux of MCPs from cosmic rays as a function of $m_{\chi}$ and $\epsilon$, in particular, closes a longstanding gap in the MCP literature as it allows to directly translate well-known bounds on an ambient MCP flux into limits on these parameters. As a result, we show that largescale underground neutrino experiments can potentially serve as the leading probe of stable dark sector particles with masses below $m_{\chi}<\frac{1}{2} m_{J / \psi} \simeq 1.5 \mathrm{GeV}$. The new limits on MCPs from Super-K are highly relevant, for example, in scenarios where MCPs constitute an SIDM component because they are (i) independent of the DM fraction made of such MCPs and (ii) probe a part of the parameter space that is inaccessible at ground-based direct-detection experiments. In addition to improvements from larger detectors, since we only consider primary meson production (providing a robust lower bound on meson production), our results can likely be further strengthened by a more detailed modeling of cosmic-ray showers.

\section{ACKNOWLEDGMENTS}

We would like to thank Dr. T.-T. Yu for a stimulating discussion of the cosmic-ray-generated light dark matter flux. We also thank Professor M. Kachelrieß, Dr. M. Smy and Professor H. Sobel for discussions. We thank Roni Harnik and Zhen Liu for providing us with the ArgoNeuT exclusion curves directly. R.P. and Y. -D. T. thank the University of Washington and the Institute for Nuclear theory for its hospitality during the final portion of this work. R. P. also thanks the Fermilab theory group for their hospitality and support. Y. -D. T. would like to thank Kavli Institute of Cosmological Physics (KICP), University of Chicago, for the hospitality and support. The work of A. K. and V. T. was supported by the U.S. Department of Energy (DOE) Grant No. DE-SC0009937. The work of A. K. was also supported by the World Premier International Research Center Initiative, MEXT Japan. The work of R. P. was supported by an the Government of Canada through an NSERC PGS-D award and by the U.S. Department of Energy, Office of Science, Office of High Energy Physics, under Award No. DE-SC0019095. This paper has been authored by Fermi Research Alliance, LLC under Contract No. DE-AC02-07CH11359 with the U.S. Department of Energy, Office of Science, Office of High Energy Physics. This work was partly performed at the Aspen Center for Physics, which is supported by National Science Foundation Grant No. PHY-1607611. Research at the Perimeter Institute was supported in part by the Government of Canada through NSERC and by the Province of Ontario through Ministry of Economic Development, Job Creation and Trade (MEDT).

\section{APPENDIX A: COSMIC-RAY PRODUCTION KINEMATICS}

\section{Boost of produced mesons}

The lab-frame energy of a meson produced in a collision, $E_{\mathfrak{m}}$, can be written as $E_{\mathfrak{m}}=\gamma_{\mathrm{cm}} \mathcal{E}+\gamma_{\mathrm{cm}} \beta_{\mathrm{cm}} \mathcal{P}_{\|}$, where curly script variables refer to center-of-mass frame quantities. We can rewrite this expression in terms of $x_{F}=\mathcal{P}_{\|} / p_{\text {max }}$ ("Feynman-X"), where $p_{\max }=\frac{1}{2} \sqrt{s}\left(1-m_{\mathfrak{m}}^{2} / s\right)$ is the largest possible longitudinal momentum allowed by kinematic constraints; $x_{F}$ therefore varies from -1 (backward pointing) to +1 (forward pointing). Written in terms of $x_{F}$, our formula is given by $E_{\mathfrak{m}}=\gamma_{\mathrm{cm}} p_{\max }\left(\mathcal{E} / p_{\max }+\beta_{\mathrm{cm}} x_{F}\right)$, or

$\gamma_{\mathfrak{m}}=\gamma_{\mathrm{cm}} \frac{p_{\max }}{m_{\mathfrak{m}}}\left(\sqrt{x_{F}^{2}+\frac{p_{T}^{2}}{p_{\max }^{2}}+\frac{m_{\mathfrak{m}}^{2}}{p_{\max }^{2}}}+\beta_{\mathrm{cm}} x_{F}\right)$.

Since $p_{T}=\mathcal{P}_{T} \ll p_{\max }$, we can neglect it in our analysis. Therefore, we can obtain $\gamma_{\mathfrak{m}}\left(x_{F}\right)$ from 


$$
\gamma_{\mathfrak{m}} \approx \gamma_{\mathrm{cm}} \frac{p_{\max }}{m_{\mathfrak{m}}}\left(\sqrt{x_{F}^{2}+\frac{m_{\mathfrak{m}}^{2}}{p_{\max }^{2}}}+\beta_{\mathrm{cm}} x_{F}\right) .
$$

This equation can be inverted to yield two branches $x_{F}^{( \pm)}\left(\gamma_{\mathfrak{m}}\right)$,

$$
x_{F}^{( \pm)}=-\gamma_{\mathrm{cm}} \gamma_{\mathfrak{m}}\left(\beta_{\mathrm{cm}} \pm \beta_{\mathfrak{m}}\right) \frac{m_{\mathfrak{m}}}{p_{\max }},
$$

corresponding to the two solutions of the quadratic equation.

\section{Meson decay to millicharged particles}

Most of the decay modes we consider involve two-body final states. For example, in the case of the $J / \psi$, the differential decay in the rest frame of the parent meson is monoenergetic $\mathrm{d} \Gamma / \mathrm{d} \mathcal{E}_{\chi} \propto \delta\left(\mathcal{E}_{\chi}-\frac{1}{2} m_{J / \psi}\right)$ (in this subsection, curly letters refer to meson rest-frame quantities). Upon boosting to the lab frame, this becomes a box distribution, $\operatorname{Box}\left(E_{\chi} \mid \gamma_{\mathfrak{m}}\right)$, of width $E_{\chi}^{(+)}-E_{\chi}^{(-)}$and height $1 /\left(E_{\chi}^{(+)}-E_{\chi}^{(-)}\right)$, where

$$
E_{\chi}^{( \pm)}=\gamma_{J / \psi}\left(\mathcal{E}_{\chi} \pm \beta_{J / \psi} \mathcal{P}_{\chi}\right) .
$$

Equivalently, in terms of the MCP's lab-frame boosts, we have

$$
\gamma_{\chi}^{( \pm)}=\gamma_{J / \psi} \tilde{\gamma}_{\chi}\left(1 \pm \beta_{J / \psi} \tilde{\beta}_{\chi}\right)
$$

where $\tilde{\gamma}_{\chi}$ and $\tilde{\beta}_{\chi}$ are the boost and velocity of the MCP in the meson rest frame. In the case of $\rho^{0}$ and $\phi$, the dominant decay mode is also a two body final state (e.g., $\rho^{0} \rightarrow \chi \bar{\chi}$ ). For $\omega$, the SM branching ratio for $\omega \rightarrow \pi^{0} \ell^{+} \ell^{-}$is roughly 10 times larger than $\omega \rightarrow \ell^{+} \ell^{-}$[89], but this decay mode is only accessible for $m_{\chi} \leq \frac{1}{2}\left(m_{\omega}-m_{\pi}\right) \approx 325 \mathrm{MeV}$, as opposed to $m_{\chi} \leq \frac{1}{2} m_{\omega} \approx 390 \mathrm{MeV}$ for the direct two body decay. We therefore neglect this decay mode ${ }^{5}$ which will underestimate the MCP flux by a factor of $\sim \mathrm{O}$ (few) in the window $275 \mathrm{MeV} \gtrsim m_{\chi} \gtrsim 325 \mathrm{MeV}$ and focus instead on $\omega \rightarrow \chi \bar{\chi}$. The branching ratio for MCPs can be obtained by a simple rescaling of the di-muon branching ratio

$\operatorname{BR}(\mathfrak{m} \rightarrow \chi \bar{\chi})=\epsilon^{2} \sqrt{\frac{m_{\mathfrak{m}}^{2}-4 m_{\chi}^{2}}{m_{\mathfrak{m}}^{2}-4 m_{\mu}^{2}}} \operatorname{BR}\left(\mathfrak{m} \rightarrow \mu^{+} \mu^{-}\right)$,

where $\operatorname{BR}\left(\rho^{0} \rightarrow \mu^{+} \mu^{-}\right)=4.55 \times 10^{-5}, \operatorname{BR}\left(\omega \rightarrow \mu^{+} \mu^{-}\right)=$ $7.4 \times 10^{-5}$, and $\operatorname{BR}\left(\phi \rightarrow \mu^{+} \mu^{-}\right)=2.87 \times 10^{-4}$ [89].

For the Dalitz decay $\eta \rightarrow \gamma \chi \bar{\chi}$, the MCPs are not monoenergetic in the meson rest frame. Nevertheless, each infinitesimal rest frame energy $\mathcal{E}_{\chi}$ can be treated as

\footnotetext{
${ }^{5}$ Including $\omega \rightarrow \pi^{0} \chi \bar{\chi}$ would involve a chiral perturbation theory calculation analogous to the one performed for $\eta \rightarrow \gamma \chi \bar{\chi}$.
}

described above provided we integrate over all such $\mathcal{E}_{\chi}$, weighted by the differential decay rate. Therefore, the labframe distribution of MCPs from $\eta$ decay is given by

$$
\left[\frac{1}{\Gamma_{\eta}} \frac{\mathrm{d} \Gamma_{\eta}}{\mathrm{d} E_{\chi}}\right]_{\mathrm{lab}}=\int \mathrm{d} \mathcal{E}_{\chi}\left[\frac{1}{\Gamma_{\eta}} \frac{\mathrm{d} \Gamma_{\eta}}{\mathrm{d} \mathcal{E}_{\chi}}\right]_{\mathrm{rest}} \times \operatorname{Box}\left(E_{\chi} \mid \gamma_{\mathfrak{m}}\right),
$$

where $\Gamma_{\eta}=\Gamma(\eta \rightarrow \chi \bar{\chi} \gamma)$. From Eq. (A7), $P\left(\gamma_{\chi} \mid \gamma_{\mathfrak{m}}\right)$ is readily obtained using

$$
P\left(\gamma_{\chi} \mid \gamma_{\mathfrak{m}}\right)=\left[\frac{1}{\Gamma} \frac{\mathrm{d} \Gamma}{\mathrm{d} \gamma_{\chi}}\right]_{\mathrm{lab}}
$$

and the chain rule. We neglect the $\eta$ form factor and compute $\frac{1}{\Gamma}[\mathrm{d} \Gamma / \mathrm{d} \mathcal{E}]$ using the Wess-Zumino $\gamma \gamma P$ vertex, with $P$ as pseudoscalar meson [106,107].

\section{APPENDIX B: ATMOSPHERIC MESON PRODUCTION RATE}

Our treatment of meson production in the upper atmosphere is data driven and centers mostly around the ratio of $\sigma(p p \rightarrow \mathfrak{m} X) / \sigma_{\text {inel }}(p p)$ which varies as a function of center-of-mass energy. Although we have tried to inform our fits using data across a wide range of center-of-mass energies (or equivalently $\gamma_{\mathrm{cm}}$ ), there is a limited window of "important" center-of-mass boosts that is determined by the competition between a rising inclusive cross section and a sharply falling cosmic-ray flux as a function of $\gamma_{\mathrm{cm}}$ (the typical $I_{\mathrm{CR}} \sim E^{-2.7}$ scaling translates to roughly $\left.\mathcal{I}_{\mathrm{CR}} \sim \gamma_{\mathrm{cm}}^{-4.5}\right)$. This is illustrated in Fig. 3 of the main text where one can see that the relevant ranges are $\gamma_{\mathrm{cm}}$ between 1.5-5 for $\eta$ mesons, between 1.5 and 10 for $\rho$ (and $\omega$ ) and $\phi$, and between 3 and 25 for $J / \psi$.

The rest of this section is devoted to our parametrization of the available inclusive cross section data, which we separate into a discussion of $\sigma_{\mathfrak{m}}\left(\gamma_{\mathrm{cm}}\right)$ and $P\left(\gamma_{\mathfrak{m}} \mid \gamma_{\mathrm{cm}}\right)$. It is important to note that although $P\left(\gamma_{\mathfrak{m}} \mid \gamma_{\mathrm{cm}}\right)$ is poorly constrained by the data, we were able to find that its impact on our sensitivity curves is marginal; this is because the total number of MCPs produced is independent of this quantity. In contrast, although it has a relatively comprehensive data set, the production cross section $\sigma_{\mathfrak{m}}\left(\gamma_{\mathrm{cm}}\right)$ in the window of maximal production (as shown in Fig. 3 of the main text) can have a substantial impact on the MCP signal (bounds

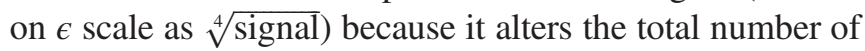
MCPs produced. We therefore anticipate that the uncertainties in the production cross section are the dominant source of error in our analysis [at the level of $\sim O$ (few)].

\section{1. $\eta$ mesons}

Eta meson production in $p p$ collisions has been most extensively measured in the near-threshold regime for the exclusive process $p p \rightarrow \eta p p[95,97]$. Near threshold this is the only available channel, such that this cross section can 
be taken as a reasonable estimate of the total inclusive cross section. Further away from threshold bona fide measurements of the inclusive cross section are scarcer, but we have identified four measurements in the literature at $\sqrt{s}=3.17$, 27.45, 38.8, and $53 \mathrm{GeV}$ [92-94,96,97]. We split the available data into two subsets, near-threshold exclusive production (defined as $p p \rightarrow p p \eta$ measurements for $\sqrt{s} \leq 3 \mathrm{GeV}$ ) and far-from-threshold inclusive data (defined as $p p \rightarrow \eta X$ for $\sqrt{s}>3 \mathrm{GeV}$ ). We fit the near-threshold data for $\sigma_{\eta}(\sqrt{s})$ with the function $f(x)=a(x-2.42)^{b} x^{c}$ where $x \equiv \sqrt{s_{p p} / \mathrm{GeV}}$. For the far-from-threshold data, we instead use $g(x)=a(1+|b| /$ $\left.(x-2.42)^{2}\right) \log ^{2}(x)$. In both cases, a weighted linear regression to the data was performed. Using the best fit values for both fits, and demanding that the function is continuous, we find

$$
\begin{aligned}
\sigma_{\eta}\left(\gamma_{\mathrm{cm}}\right)= & \Theta\left(\gamma_{\mathrm{cm}}-\gamma^{\prime}\right) f\left(1.876 \gamma_{\mathrm{cm}}\right) \\
& +\Theta\left(\gamma^{\prime}-\gamma_{\mathrm{cm}}\right) g\left(1.876 \gamma_{\mathrm{cm}}\right),
\end{aligned}
$$

where the numerical factor comes from the relationship $\sqrt{s}=2 m_{p} \gamma_{\mathrm{cm}}=(1.876 \mathrm{GeV}) \gamma_{\mathrm{cm}}$. The functions $f(x)$ and $g(x)$, with their best fit values, are given by

$$
\begin{array}{r}
f(x)=(0.0176 \mathrm{mb}) \times(x-2.42)^{2.22} x^{4.59}, \\
g(x)=(1.32 \mathrm{mb}) \log ^{2}(x) \times\left(1+\frac{0.356}{(x-2.42)^{2}}\right)^{-1},
\end{array}
$$

and $\gamma^{\prime}=1.59$ is chosen such that Eq. (B1) is continuous; the fit is shown versus the data (with error bars when available) in Fig. 7.

For the differential cross section $\mathrm{d} \sigma_{\mathfrak{m}} / \mathrm{d} x_{F}$, measurements at NA27 [94] strongly suggest an exponential distribution,

$$
\frac{\mathrm{d} \sigma_{\eta}}{\mathrm{d} x_{F}}=\sigma_{\eta} \times \frac{c_{\eta} / 2}{1-\exp \left[-c_{\eta}\right]} \exp \left[-c_{\eta}\left|x_{F}\right|\right],
$$

where $c_{\eta}$ depends on $\gamma_{\mathrm{cm}}$. Measurements from NA27 at $\sqrt{s}=27.5 \mathrm{GeV}$ (corresponding to $\gamma_{\mathrm{cm}}=14.6$ ) fix $c_{\eta} \approx 9.5$ [94]. One generally expects that $c_{\eta}$ will be a monotonically increasing function of $\gamma_{\mathrm{cm}}$ and that $c_{\eta}>0$. The simplest functional form that satisfies these expectations and agrees with the measurement of [94] is

$$
c_{\eta}\left(\gamma_{\mathrm{cm}}\right)=9.5+(\text { slope }) \times\left(\gamma_{\mathrm{cm}}-14.6\right) .
$$

We take slope $\approx \frac{1}{2}$. We checked that our sensitivity to MCPs from experiments such as SK is relatively insensitive to the value of the slope parameter.

\section{Light vector mesons}

The production cross section for the $\rho^{0}$ meson is relatively well measured $[93,95,97]$. Like the $\eta$ meson, we perform a best fit analysis with the function $g(x)$, but

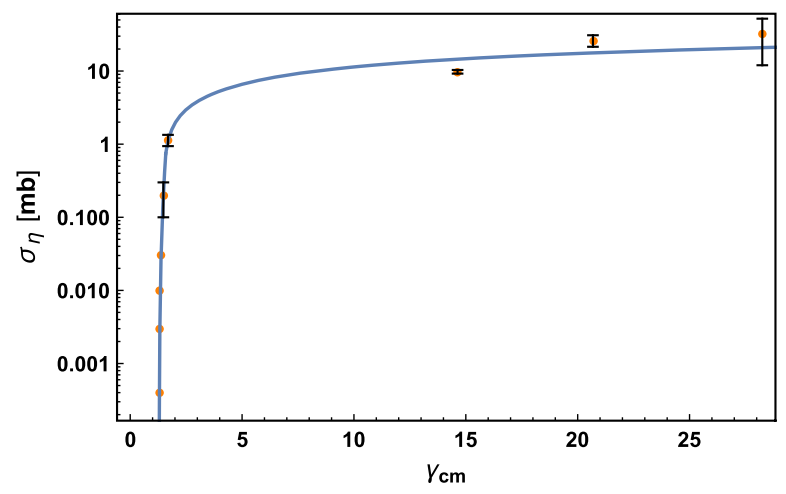

FIG. 7. Production cross section for $p p \rightarrow \eta X$ as a function of $\gamma_{\mathrm{cm}}=\frac{1}{2} \sqrt{s} / m_{p}$. The data are taken from Refs. [92-97] and fitted using the piecewise procedure described in the text; the smooth curve is Eq. (B1).

without weighted errors. We find the data to be reasonably well described by

$$
\begin{aligned}
\sigma_{\rho}\left(\gamma_{\mathrm{cm}}\right) \approx & (1.35 \mathrm{mb}) \log ^{2}\left(1.876 \gamma_{\mathrm{cm}}\right) \\
& \times\left(1+\frac{13.4}{\left(1.876 \gamma_{\mathrm{cm}}-2.61\right)^{2}}\right)^{-1}
\end{aligned}
$$

A comparison between the available data and our smooth fit is shown in Fig. 8.

We found that the data for $\sigma(p p \rightarrow \rho X)$ had a much better coverage than the corresponding $\omega$ production cross section and where there are measurements of the $\omega$ cross section it is nearly identical to the $\rho$ cross section. We therefore estimated the $\omega$ cross section $\sigma_{\omega}\left(\gamma_{\omega}\right) \simeq \sigma_{\rho}\left(\gamma_{\rho}\right)$.

For the $\phi$ meson, we find that the functional form $h(x)=$ $a\left(1+|b| /(x-2.896)^{2}\right)^{-1} x^{c}$ gives a reasonable fit to the data $[93,95,97]$. After an unweighted regression, we find that $\sigma_{\phi}$ is well described, cf. Fig. 9, by

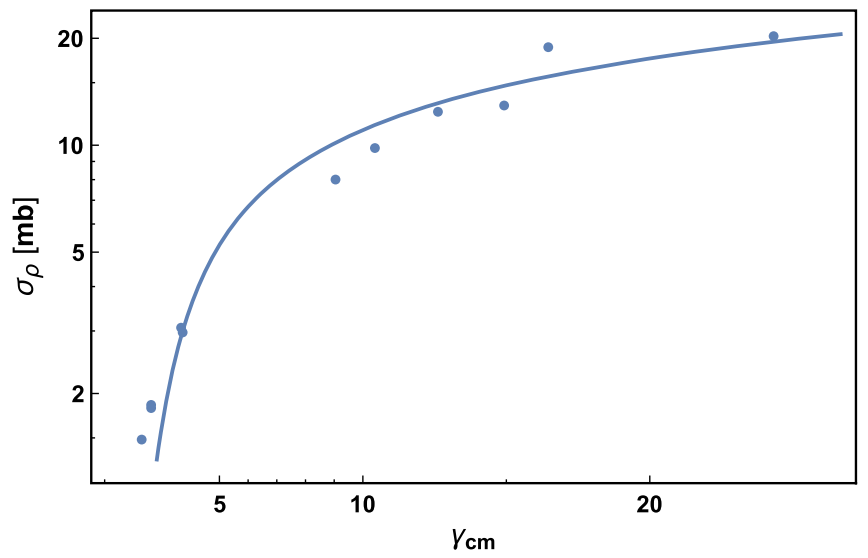

FIG. 8. Compilation of $p p \rightarrow \rho X$ cross sections as a function of $\gamma_{\mathrm{cm}}$ taken from $[93,95,97]$. 


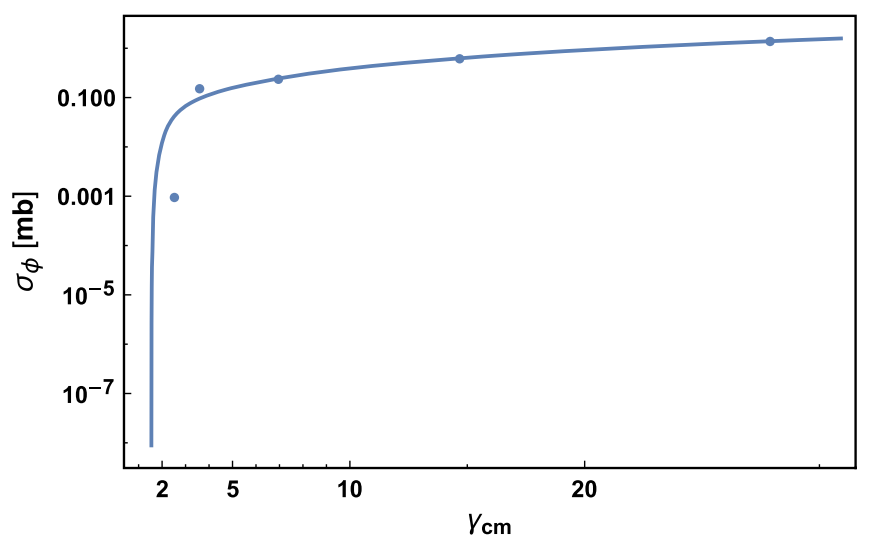

FIG. 9. Compilation of $p p \rightarrow \phi X$ cross sections as a function of $\gamma_{\mathrm{cm}}$ taken from [93-95].

$$
\begin{aligned}
\sigma_{\phi}\left(\gamma_{\mathrm{cm}}\right)= & (0.01 \mathrm{mb})\left(1.876 \gamma_{\mathrm{cm}}\right)^{1.23} \\
& \times\left(1+\frac{2.4}{\left(1.876 \gamma_{\mathrm{cm}}-2.896\right)^{2}}\right)
\end{aligned}
$$

Like the $\eta$ meson, the longitudinal momentum distributions for the vector mesons were more difficult to find in the literature, and we rely on a single measurement at $\sqrt{s}=27.5 \mathrm{GeV}$ [94] which shows the $x_{F}$ dependence to be described by Eq. (B4) with $c_{V}=c_{\rho}=c_{\omega}=c_{\phi} \approx 7.7$. We expect this value to be smaller at lower center-of-mass energies and so take

$$
c_{V}=7.7+\frac{5.7}{13}\left(\gamma_{\mathrm{cm}}-14.6\right),
$$

which, just like the $c_{\eta}$, should be viewed as a cartoon of the behavior of $\mathrm{d} \sigma / \mathrm{d} x_{F}$ as a function of $\gamma_{\mathrm{cm}}$ rather than a faithful representation.

\section{3. $\mathrm{J} / \psi$ mesons}

For the $J / \psi$ mesons, we found two convenient summaries of the available data: one from E-739 (Fig. 7 in Ref. [98]) and one from HERA-B (Fig. 8 of Ref. [101]). The HERA-B compilation includes measurements at significantly higher center-of-mass energies. For comparison, we plot both sets of data in Fig. 10 where we see that the HERA-B compiled data are roughly consistent with that from the E739 paper, but suggests a steeper growth with rising center-of-mass energy. We use the best fit to the former to calculate $\sigma_{\mathfrak{m}}\left(\gamma_{\mathrm{cm}}\right)$.

For the differential distribution, we used the standard parametrization of $\mathrm{d} \sigma_{J / \psi} / \mathrm{d} x_{F}$ [100],

$$
\frac{\mathrm{d} \sigma_{J / \psi}}{\mathrm{d} x_{F}}=\sigma_{J / \psi} \times \frac{\left(c_{J / \psi}+1\right)}{2}\left(1-\left|x_{F}\right|\right)^{c_{J / \psi}} .
$$

Like $c_{\eta}$, the fit parameter $c_{J / \psi}$ depends on the center-ofmass energy, and like $c_{\eta}$ the precise value of $c_{J / \psi}$ has a

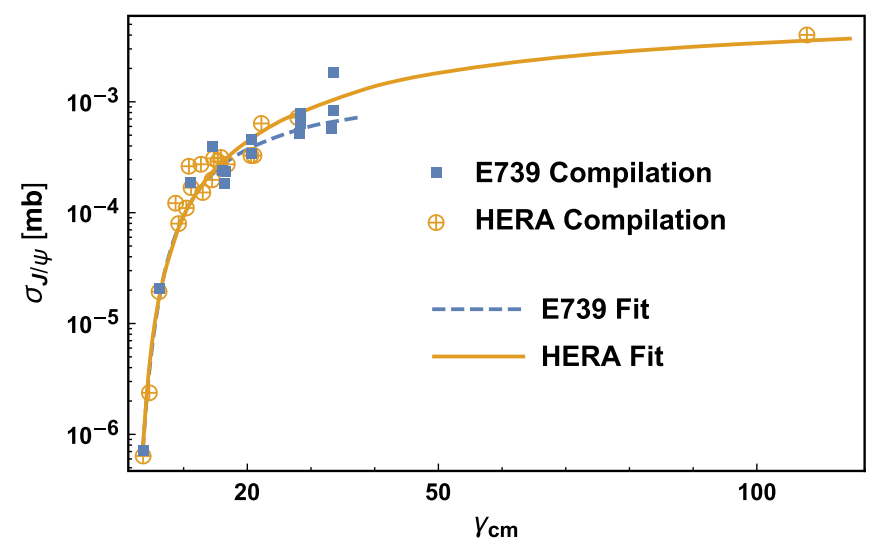

FIG. 10. Production cross section for $p p \rightarrow J / \psi X$ as a function of $\gamma_{\mathrm{cm}}=\frac{1}{2} \sqrt{s} / m_{p}$. The data points have been digitized from E-789's compilation [98] and HERA-B's compilation [101]. The solid curve is digitized from the fit presented in Ref. [101], while the dashed curve is taken from Ref. [98]. For our sensitivity analysis, we use the solid curve from [101].

relatively mild effect on the fast flux of MCPs. Data from experiments at lower energies show a preference for $c_{J / \psi} \approx$ 2 for $\sqrt{s} \leq 15 \mathrm{GeV}$ [100], whereas experiments at higher energies find larger values such as $c_{J / \Psi} \approx 6$ for $\sqrt{s} \approx$ $40 \mathrm{GeV}$ [99]; we did not find a robust set of measurements of $c_{J / \psi}$ spanning the entire range of $\gamma_{\mathrm{cm}}$ relevant for cosmicray $p p$ collisions. For simplicity, and because our final results are relatively insensitive to the details of the $x_{F}$ distribution, we take $c_{J / \psi}$ to vary linearly with $\gamma_{\mathrm{cm}}$,

$$
c_{J / \psi}=2+\frac{1}{5}\left(\gamma_{\mathrm{cm}}-5\right) \text {. }
$$

In this case, there are data at lower center-of-mass energies that suggest this formula is a reasonable interpolation.

\section{APPENDIX C: IONIZATION EXPERIMENTS}

Ionization is a very low threshold process and so we use the full flux (integrated over all boosts $\gamma_{\chi}$ ) of MCPs for ionization experiments; this corresponds to $\gamma_{\text {cut }}=1$ as shown in Fig. 4 of the main text; we denote this total flux by $\Phi\left(m_{\chi}\right)$. To translate existing bounds on an ambient MCP flux in the literature, we demand that

$$
\epsilon^{2} \times\left(\epsilon^{-2} \Phi\left(m_{\chi}\right)\right)=\Phi_{\text {ion }}(\epsilon)
$$

where $\epsilon^{-2} \Phi\left(m_{\chi}\right)$ corresponds to the $\gamma_{\text {cut }}=1$ curve in Fig. 4 of the main text (i.e., the integrated MCP flux generated in the upper atmosphere), and $\Phi_{\text {ion }}(\epsilon)$ is the joint exclusion curve obtained by combining data from MACRO $[49,50]$ and Majorana [54] as shown in Fig. 7 of [54]. We then solve for $\epsilon$ for each value of $m_{\chi}$ which determines a critical value of, $\epsilon_{c}\left(m_{\chi}\right)$, above which MCPs are excluded. 


\section{APPENDIX D: COSMOGENIC COINCIDENCE AND CUTS AT SUPER-KAMIOKANDE}

In this section, we discuss the possibility that cuts from the Super-Kamiokande Collaboration designed to limit cosmogenic activity could also remove MCP scattering events were they to occur. We conclude that this is extremely unlikely and can be neglected in our analysis.

As is clearly seen in Fig. 3, the energy of the $p p$ collision that sources an MCP can be (roughly) inferred given the meson species that was produced. Perhaps the most concerning possibility is a high-energy $p p$ collision that produces $J / \psi$ mesons (these dominate the flux for the heaviest MCPs we considered). A center-of-mass boost of $\gamma_{c} m=25$ corresponds to an incident cosmic-ray energy of $E_{p} \approx 75 \mathrm{GeV}$. Consulting Table II-28 of [108] we find that muons with energy less than $75 \mathrm{GeV}$ would penetrate only $200 \mathrm{~m}$ of water. Noting that the SK overburden is $3 \times 10^{3} \mathrm{~m}$ water equivalent, we conclude that any SM cosmic-ray particles sourced from the proton-proton collisions that produce almost all of the MCP flux would be stopped by the overburden; while it is possible that higher energy cosmic rays also produce MCPs and associated penetrating showers, this component of the flux and its contribution to the expected MCP event rate at SK are totally negligible for our analysis.

In addition to the (rather robust) energetic argument presented above, we note that while cosmic rays are collimated, they have a characteristic separation on the order of a few degrees or 0.03 radians, having two coincident events inside SK's volume within the same shower requires an angular separation of $20 \mathrm{~m}$ (detector size) $/ 5 \mathrm{~km}$ (travel length) $4 \times 10^{-3}$ radians and is hence extremely unlikely.

The final consideration that one may be worried about is coincident cosmic-ray activity from a separate high-energy muon. In the DSNB analysis, no event is allowed to be within 50 microseconds after a muon detection, requiring very strong time coincidence as well. Although it is

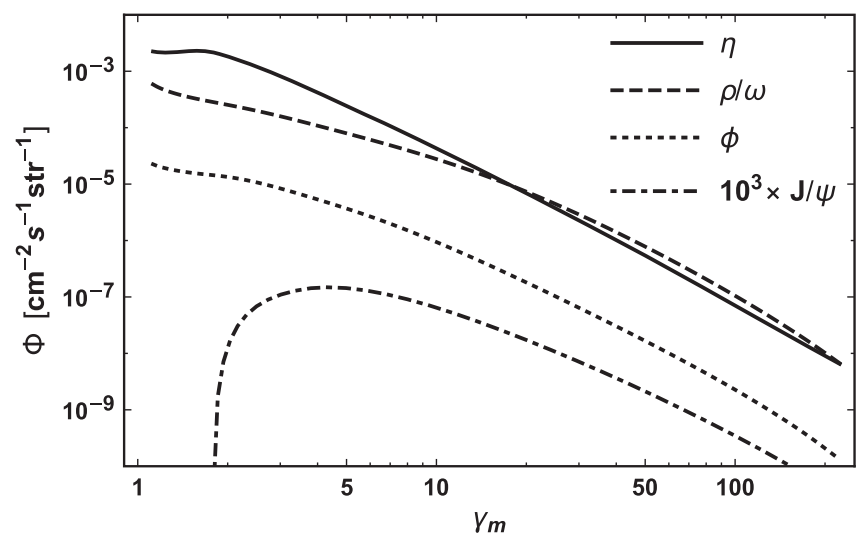

FIG. 11. Mesons fluxes from primary $p p$ collisions assuming a longitudinal momentum distribution as described in Eqs. (B4), (B5), and (B8)-(B10).

possible that a high-energy muon (i.e., with energy larger than $1 \mathrm{TeV}$ ) produced independently of an MCP could be time coincident with an MCP scattering event, this is also unlikely; the rate of muon activity in SK is $2 \mathrm{~Hz}$ such that the probability of 50 microsecond coincidence is roughly $10^{-4}$. We therefore conclude that the efficiency of the DSNB cuts for MCPs is a relatively small effect that can be safely neglected at the level of accuracy considered in our study.

\section{APPENDIX E: MESON FLUXES}

A useful biproduct of our research are the lab-frame spectra of mesons as a function of $\gamma_{\mathfrak{m}}$. Given any calculable $\mathfrak{m} \rightarrow$ dark sector decay, using the meson spectra as inputs, a flux of dark sector particles originating from primary cosmic-ray collisions can be obtained. Our results, shown in Fig. 11, rely only on simple parametrizations of the differential cross sections $\mathrm{d} \sigma / \mathrm{d} x_{F}$ and the measured production cross sections as outlined above.
[1] J. Alexander et al., Dark sectors 2016 workshop: Community report, arXiv:1608.08632.

[2] G. W. Bennett et al. (Muon g-2 Collaboration), Final report of the muon E821 anomalous magnetic moment measurement at BNL, Phys. Rev. D 73, 072003 (2006).

[3] F. Jegerlehner and A. Nyffeler, The muon g-2, Phys. Rep. 477, 1 (2009).

[4] J. P. Miller, E. de Rafael, B. L. Roberts, and D. Stöckinger, Muon (g-2): Experiment and theory, Annu. Rev. Nucl. Part. Sci. 62, 237 (2012).

[5] J. D. Bjorken, R. Essig, P. Schuster, and N. Toro, New fixed-target experiments to search for dark gauge forces, Phys. Rev. D 80, 075018 (2009).
[6] R. Essig et al., Working Group Report: New light weakly coupled particles, in Proceedings, 2013 Community Summer Study on the Future of U.S. Particle Physics: Snowmass on the Mississippi (CSS2013): Minneapolis, MN, USA, 2013 (2013).

[7] B. Batell, M. Pospelov, and A. Ritz, Exploring portals to a hidden sector through fixed targets, Phys. Rev. D 80, 095024 (2009).

[8] J. P. Lees et al. (BABAR Collaboration), Search for a Dark Photon in $e^{+} e^{-}$Collisions at BABAR, Phys. Rev. Lett. 113, 201801 (2014).

[9] R. Essig, P. Schuster, N. Toro, and B. Wojtsekhowski, An electron fixed target experiment to search for a new vector 
boson A' decaying to $e^{+} e^{-}$, J. High Energy Phys. 02 (2011) 009.

[10] A. A. Prinz et al., Search for Millicharged Particles at SLAC, Phys. Rev. Lett. 81, 1175 (1998).

[11] A. A. Prinz, The search for millicharged particles at SLAC, Ph.D. thesis, Stanford U., Phys. Dept., 2001.

[12] P. deNiverville, M. Pospelov, and A. Ritz, Observing a light dark matter beam with neutrino experiments, Phys. Rev. D 84, 075020 (2011).

[13] Y. Kahn, G. Krnjaic, J. Thaler, and M. Toups, DAEDALUS and dark matter detection, Phys. Rev. D 91, 055006 (2015).

[14] M. Pospelov and Y.-D. Tsai, Light scalars and dark photons in Borexino and LSND experiments, Phys. Lett. B 785, 288 (2018).

[15] G. Magill, R. Plestid, M. Pospelov, and Y.-D. Tsai, Dipole portal to heavy neutral leptons, Phys. Rev. D 98, 115015 (2018).

[16] G. Magill, R. Plestid, M. Pospelov, and Y.-D. Tsai, Millicharged Particles in Neutrino Experiments, Phys. Rev. Lett. 122, 071801 (2019).

[17] C. A. Argüelles, M. Hostert, and Y.-D. Tsai, Testing New Physics Explanations of MiniBooNE Anomaly at Neutrino Scattering Experiments, Phys. Rev. Lett. 123, 261801 (2019).

[18] K. J. Kelly and Y.-D. Tsai, Proton fixed-target scintillation experiment to search for millicharged dark matter, Phys. Rev. D 100, 015043 (2019).

[19] C. A. Argüelles et al., White paper on new opportunities at the next-generation neutrino experiments (Part 1: BSM neutrino physics and dark matter), arXiv:1907.08311.

[20] Y.-D. Tsai, P. deNiverville, and M.X. Liu, The highenergy frontier of the intensity frontier: Closing the dark photon, inelastic dark matter, and muon g-2 windows, arXiv: 1908.07525.

[21] S. Davidson, S. Hannestad, and G. Raffelt, Updated bounds on millicharged particles, J. High Energy Phys. 05 (2000) 003.

[22] A. Ball et al., A letter of intent to install a milli-charged particle detector at LHC P5, arXiv:1607.04669.

[23] A. Haas, C. S. Hill, E. Izaguirre, and I. Yavin, Looking for milli-charged particles with a new experiment at the LHC, Phys. Lett. B 746, 117 (2015).

[24] A. Kusenko, S. Pascoli, and D. Semikoz, New bounds on $\mathrm{MeV}$ sterile neutrinos based on the accelerator and SuperKamiokande results, J. High Energy Phys. 11 (2005) 028.

[25] P.-f. Yin and S.-h. Zhu, Detecting light long-lived particle produced by cosmic ray, Phys. Lett. B 685, 128 (2010).

[26] P.-K. Hu, A. Kusenko, and V. Takhistov, Dark cosmic rays, Phys. Lett. B 768, 18 (2017).

[27] T. Bringmann and M. Pospelov, Novel Direct Detection Constraints on Light Dark Matter, Phys. Rev. Lett. 122, 171801 (2019).

[28] C. Argüelles, P. Coloma, P. Hernández, and V. Muñoz, Searches for atmospheric long-lived particles, J. High Energy Phys. 02 (2020) 190.

[29] P. Coloma, P. Hernández, V. Muñoz, and I. M. Shoemaker, New constraints on heavy neutral leptons from SuperKamiokande data, Eur. Phys. J. C 80, 235 (2020).
[30] J. Alvey, M. Campos, M. Fairbairn, and T. You, Light Dark Matter from Inelastic Cosmic Ray Collisions, Phys. Rev. Lett. 123, 261802 (2019); Erratum, Phys. Rev. Lett. 123, 261802 (2019).

[31] K. Bondarenko, A. Boyarsky, T. Bringmann, M. Hufnagel, K. Schmidt-Hoberg, and A. Sokolenko, Direct detection and complementary constraints for sub-GeV dark matter, J. High Energy Phys. 03 (2020) 118.

[32] C. Cappiello and J. F. Beacom, Strong new limits on light dark matter from neutrino experiments, Phys. Rev. D 100, 103011 (2019).

[33] J. B. Dent, B. Dutta, J. L. Newstead, and I. M. Shoemaker, Bounds on cosmic ray-boosted dark matter in simplified models and its corresponding neutrino-floor, Phys. Rev. D 101, 116007 (2020).

[34] Y. Ema, F. Sala, and R. Sato, Light Dark Matter at Neutrino Experiments, Phys. Rev. Lett. 122, 181802 (2019).

[35] C. M. G. Lattes, H. Muirhead, G. P. S. Occhialini, and C. F. Powell, Processes involving charged mesons, Nature (London) 159, 694 (1947).

[36] S. H. Neddermeyer and C. D. Anderson, Note on the nature of cosmic ray particles, Phys. Rev. 51, 884 (1937).

[37] R. Abbasi et al. (IceCube Collaboration), The IceCube data acquisition system: Signal capture, digitization, and timestamping, Nucl. Instrum. Methods Phys. Res., Sect. A 601, 294 (2009).

[38] K. Abe et al., Calibration of the Super-Kamiokande detector, Nucl. Instrum. Methods Phys. Res., Sect. A 737, 253 (2014).

[39] K. Abe et al. (Hyper-Kamiokande Collaboration), HyperKamiokande design report, arXiv:1805.04163.

[40] Z. Djurcic et al. (JUNO Collaboration), JUNO conceptual design report, arXiv:1508.07166.

[41] B. Abi et al. (DUNE Collaboration), The DUNE far detector interim design report volume 1: Physics, technology and strategies, arXiv:1807.10334.

[42] P.F. Smith, Searches for fractional electric charge in terrestrial materials, Annu. Rev. Nucl. Part. Sci. 39, 73 (1989); 39, 527 (1987).

[43] M. I. Dobroliubov and A. Yu. Ignatiev, Millicharged Particles, Phys. Rev. Lett. 65, 679 (1990).

[44] E. Golowich and R. W. Robinett, Limits on millicharged matter from beam dump experiments, Phys. Rev. D 35, 391 (1987).

[45] K. S. Babu, T. M. Gould, and I. Z. Rothstein, Closing the windows on MeV Tau neutrinos, Phys. Lett. B 321, 140 (1994).

[46] S. N. Gninenko, N. V. Krasnikov, and A. Rubbia, Search for millicharged particles in reactor neutrino experiments: A probe of the PVLAS anomaly, Phys. Rev. D 75, 075014 (2007).

[47] L. Chuzhoy and E. W. Kolb, Reopening the window on charged dark matter, J. Cosmol. Astropart. Phys. 07 (2009) 014.

[48] D. Dunsky, L. J. Hall, and K. Harigaya, CHAMP cosmic rays, J. Cosmol. Astropart. Phys. 07 (2019) 015.

[49] M. Ambrosio et al. (MACRO Collaboration), A search for lightly ionizing particles with the MACRO detector, Phys. Rev. D 62, 052003 (2000). 
[50] M. Ambrosio et al. (MACRO Collaboration), Final search for lightly ionizing particles with the MACRO detector, arXiv:hep-ex/0402006.

[51] M. Mori et al. (Kamiokande-II Collaboration), Search for fractionally charged particles in Kamiokande-II, Phys. Rev. D 43, 2843 (1991).

[52] M. Aglietta et al., Search for fractionally charged particles in the Mont Blanc LSD scintillation detector, Astropart. Phys. 2, 29 (1994).

[53] R. Agnese et al. (CDMS Collaboration), First Direct Limits on Lightly Ionizing Particles with Electric Charge Less Than e/6, Phys. Rev. Lett. 114, 111302 (2015).

[54] S. I. Alvis et al. (Majorana Collaboration), First Limit on the Direct Detection of Lightly Ionizing Particles for Electric Charge as Low as $e / 1000$ with the Majorana Demonstrator, Phys. Rev. Lett. 120, 211804 (2018).

[55] K. Bays et al. (Super-Kamiokande Collaboration), Supernova relic neutrino search at Super-Kamiokande, Phys. Rev. D 85, 052007 (2012).

[56] J. D. Bowman, A. E. E. Rogers, R. A. Monsalve, T. J. Mozdzen, and N. Mahesh, An absorption profile centered at 78 megahertz in the sky-averaged spectrum, Nature (London) 555, 67 (2018).

[57] J. B. Muñoz and A. Loeb, A small amount of mini-charged dark matter could cool the baryons in the early Universe, Nature (London) 557, 684 (2018).

[58] R. Barkana, N. J. Outmezguine, D. Redigolo, and T. Volansky, Strong constraints on light dark matter interpretation of the EDGES signal, Phys. Rev. D 98, 103005 (2018).

[59] H. Liu, N. J. Outmezguine, D. Redigolo, and T. Volansky, Reviving millicharged dark matter for 21-cm cosmology, Phys. Rev. D 100, 123011 (2019).

[60] A. A. Aguilar-Arevalo et al. (MiniBooNE DM Collaboration), Dark matter search in nucleon, pion, and electron channels from a proton beam dump with MiniBooNE, Phys. Rev. D 98, 112004 (2018).

[61] R. Acciarri et al. (ArgoNeuT Collaboration), Improved Limits on Millicharged Particles Using the ArgoNeuT Experiment at Fermilab, Phys. Rev. Lett. 124, 131801 (2020).

[62] S. Chatrchyan et al. (CMS Collaboration), Search for fractionally charged particles in $p p$ collisions at $\sqrt{s}=7 \mathrm{TeV}$, Phys. Rev. D 87, 092008 (2013).

[63] H. Vogel and J. Redondo, Dark radiation constraints on minicharged particles in models with a hidden photon, J. Cosmol. Astropart. Phys. 02 (2014) 029.

[64] J. Jaeckel, M. Jankowiak, and M. Spannowsky, LHC probes the hidden sector, Phys. Dark Universe 2, 111 (2013).

[65] B. Holdom, Two U(1)'s and epsilon charge shifts, Phys. Lett. 166B, 196 (1986).

[66] P. A. M. Dirac, Quantized singularities in the electromagnetic field, Proc. R. Soc. A 133, 133, 278 (1931).

[67] J. C. Pati and A. Salam, Unified lepton-hadron symmetry and a gauge theory of the basic interactions, Phys. Rev. D 8, 1240 (1973).

[68] H. Georgi and S. L. Glashow, Unity of All Elementary Particle Forces, Phys. Rev. Lett. 32, 438 (1974).
[69] H. Georgi, The state of the art-gauge theories, AIP Conf. Proc. 23, 575 (1975).

[70] H. Fritzsch and P. Minkowski, Unified interactions of leptons and hadrons, Ann. Phys. (N.Y.) 93, 193 (1975).

[71] G. Shiu, P. Soler, and F. Ye, Milli-Charged Dark Matter in Quantum Gravity and String Theory, Phys. Rev. Lett. 110, 241304 (2013).

[72] N. Arkani-Hamed, D. P. Finkbeiner, T. R. Slatyer, and N. Weiner, A theory of dark matter, Phys. Rev. D 79, 015014 (2009).

[73] M. Pospelov, Secluded U(1) below the weak scale, Phys. Rev. D 80, 095002 (2009).

[74] P. Agrawal, F.-Y. Cyr-Racine, L. Randall, and J. Scholtz, Make dark matter charged again, J. Cosmol. Astropart. Phys. 05 (2017) 022.

[75] N. Kurinsky, D. Baxter, Y. Kahn, and G. Krnjaic, Dark matter interpretation of excesses in multiple direct detection experiments, Phys. Rev. D 102, 015017 (2020).

[76] A. E. Robinson and É. Michaud, Comment on a dark matter interpretation of excesses in multiple direct detection experiments, Phys. Rev. D 102, 015017 (2020); arXiv:2002.08893.

[77] H. Goldberg and L. J. Hall, A new candidate for dark matter, Phys. Lett. B 174, 151 (1986).

[78] T. Emken, R. Essig, C. Kouvaris, and M. Sholapurkar, Direct detection of strongly interacting sub-GeV dark matter via electron recoils, J. Cosmol. Astropart. Phys. 09 (2019) 070.

[79] G. D. Starkman, A. Gould, R. Esmailzadeh, and S. Dimopoulos, Opening the window on strongly interacting dark matter, Phys. Rev. D 41, 3594 (1990).

[80] S. L. Dubovsky, D. S. Gorbunov, and G. I. Rubtsov, Narrowing the window for millicharged particles by CMB anisotropy, Pis'ma Zh. Eksp. Teor. Fiz. 79, 3 (2004) [JETP Lett. 79, 1 (2004)].

[81] A. D. Dolgov, S. L. Dubovsky, G. I. Rubtsov, and I. I. Tkachev, Constraints on millicharged particles from Planck data, Phys. Rev. D 88, 117701 (2013).

[82] E. D. Kovetz, V. Poulin, V. Gluscevic, K. K. Boddy, R. Barkana, and M. Kamionkowski, Tighter limits on dark matter explanations of the anomalous EDGES $21 \mathrm{~cm}$ signal, Phys. Rev. D 98, 103529 (2018).

[83] D. Green et al., Messengers from the early Universe: Cosmic neutrinos and other light relics, arXiv:1903.04763.

[84] A. Berlin, D. Hooper, G. Krnjaic, and S. D. McDermott, Severely Constraining Dark Matter Interpretations of the 21-cm Anomaly, Phys. Rev. Lett. 121, 011102 (2018).

[85] J. Rich, R. Rocchia, and M. Spiro, A search for strongly interacting dark matter, Phys. Lett. B 194, 173 (1987); 194, 173 (1987).

[86] A. L. Erickcek, P. J. Steinhardt, D. McCammon, and P. C. McGuire, Constraints on the interactions between dark matter and baryons from the x-ray quantum calorimetry experiment, Phys. Rev. D 76, 042007 (2007).

[87] M. S. Mahdawi and G. R. Farrar, Constraints on dark matter with a moderately large and velocity-dependent DM-nucleon cross-section, J. Cosmol. Astropart. Phys. 10 (2018) 007. 
[88] J.-T. Li and T. Lin, Dynamics of millicharged dark matter in supernova remnants, Phys. Rev. D 101, 103034 (2020).

[89] M. Tanabashi et al. (Particle Data Group), Review of particle physics, Phys. Rev. D 98, 030001 (2018).

[90] T. Bringmann, J. Edsjö, P. Gondolo, P. Ullio, and L. Bergström, DarkSUSY 6: An advanced tool to compute dark matter properties numerically, J. Cosmol. Astropart. Phys. 07 (2018) 033.

[91] M. J. Boschini et al., Solution of heliospheric propagation: Unveiling the local interstellar spectra of cosmic ray species, Astrophys. J. 840, 115 (2017).

[92] G. Jancso et al., Evidence for dominant vector meson production in inelastic proton proton collisions at $53-\mathrm{GeV}$ center-of-mass energy, Nucl. Phys. B124, 1 (1977).

[93] A. Baldini, V. Flaminio, W. G. Moorhead, and Douglas R. O. Morrison, in Total Cross-Sections for Reactions of High Energy Particles (Including Elastic, Topological, Inclusive and Exclusive Reactions)/Totale Wirkungsquerschnitte für Reaktionen Hochenergetischer Teilchen (Einschließlich Elastischer, Topologischer, Inklusive, edited by H. Schopper, Landolt-Boernstein-Group I Elementary Particles, Nuclei and Atoms, Vol. 12b (Springer, New York, 1988).

[94] M. Aguilar-Benitez et al., Inclusive particle production in 400-GeV/c p p interactions, Z. Phys. C 50, 405 (1991).

[95] A. Sibirtsev, W. Cassing, and U. Mosel, Heavy meson production in proton-nucleus reactions with empirical spectral functions, Z. Phys. A 358, 357 (1997).

[96] P. M. Hanlet, Inclusive measurements of the eta cross section at $38.8 \mathrm{GEV}$ and the branching ratio for eta meson going to positive muon negative muon, Ph.D. thesis, University of Virginia, 1995.

[97] G. Agakishiev et al. (HADES Collaboration), Inclusive dielectron spectra in $p+p$ collisions at $3.5 \mathrm{GeV}$, Eur. Phys. J. A 48, 64 (2012).
[98] D. M. Kaplan (E789 Collaboration), Charmonium production in Fermilab E789, Int. J. Mod. Phys. A 12, 3827 (1997).

[99] T. Alexopoulos et al. (E-771 Collaboration), Differential cross-sections of $J / \psi$ and $\psi^{\prime}$ in $800-\mathrm{GeV} / \mathrm{c}$ p-Si interactions, Phys. Rev. D 55, 3927 (1997).

[100] R. Vogt, $J / \psi$ production and suppression, Phys. Rep. 310, 197 (1999).

[101] I. Abt et al. (HERA-B Collaboration), Measurement of the $J / \psi$ production cross section in $920-\mathrm{GeV} / \mathrm{c}$ fixed-target proton-nucleus interactions, Phys. Lett. B 638, 407 (2006).

[102] L. B. Auerbach et al. (LSND Collaboration), Measurement of electron-neutrino-electron elastic scattering, Phys. Rev. D 63, 112001 (2001).

[103] N. Abgrall et al. (LEGEND Collaboration), The large enriched germanium experiment for neutrinoless double beta decay (LEGEND), AIP Conf. Proc. 1894, 020027 (2017).

[104] J. F. Beacom and M. R. Vagins, GADZOOKS! AntiNeutrino Spectroscopy with Large Water Cherenkov Detectors, Phys. Rev. Lett. 93, 171101 (2004).

[105] K. Møller, A. M. Suliga, I. Tamborra, and P. B. Denton, Measuring the supernova unknowns at the next-generation neutrino telescopes through the diffuse neutrino background, J. Cosmol. Astropart. Phys. 05 (2018) 066.

[106] R. D. Pisarski, T. L. Trueman, and M. H. G. Tytgat, How $\pi^{0} \rightarrow \gamma \gamma$ changes with temperature, Phys. Rev. D 56, 7077 (1997).

[107] J. Wess and B. Zumino, Consequences of anomalous ward identities, Phys. Lett. 37B, 95 (1971).

[108] D. E. Groom, N. V. Mokhov, and S. I. Striganov, Muon stopping power and range tables $10-\mathrm{MeV}$ to $100-\mathrm{TeV}$, At. Data Nucl. Data Tables 78, 183 (2001). 